\title{
Stromal alterations in ovarian cancers via wavelength dependent Second Harmonic Generation microscopy and optical scattering
}

Karissa B. Tilbury ${ }^{1}$, Kirby R. Campbell ${ }^{1}$, Kevin W. Eliceiri ${ }^{1,2,3}$, Sana M. Salih ${ }^{4}$, Manish Patankar ${ }^{4}$ and Paul J. Campagnola ${ }^{1,2^{*}}$

\begin{abstract}
Background: Ovarian cancer remains the most deadly gynecological cancer with a poor aggregate survival rate; however, the specific rates are highly dependent on the stage of the disease upon diagnosis. Current screening and imaging tools are insufficient to detect early lesions and are not capable of differentiating the subtypes of ovarian cancer that may benefit from specific treatments.

Method: As an alternative to current screening and imaging tools, we utilized wavelength dependent collagen-specific Second Harmonic Generation (SHG) imaging microscopy and optical scattering measurements to probe the structural differences in the extracellular matrix (ECM) of normal stroma, benign tumors, endometrioid tumors, and low and high-grade serous tumors.

Results: The SHG signatures of the emission directionality and conversion efficiency as well as the optical scattering are related to the organization of collagen on the sub-micron size scale and encode structural information. The wavelength dependence of these readouts adds additional characterization of the size and distribution of collagen fibrils/fibers relative to the interrogating wavelengths. We found a strong wavelength dependence of these metrics that are related to significant structural differences in the collagen organization and are consistent with the dualistic classification of type I and II serous tumors. Moreover, type I endometrioid tumors have strongly differing ECM architecture than the serous malignancies. The SHG metrics and optical scattering measurements were used to form a linear discriminant model to classify the tissues, and we obtained high accuracy (>90\%) between high-grade serous tumors from the other tissue types. High-grade serous tumors account for $~ 70 \%$ of ovarian cancers, and this delineation has potential clinical applications in terms of supplementing histological analysis, understanding the etiology, as well as development of an in vivo screening tool.

(Continued on next page)
\end{abstract}

\footnotetext{
* Correspondence: pcampagnola@wisc.edu

${ }^{1}$ Laboratory for Optical and Computational Instrumentation, Department of

Biomedical Engineering, University of Wisconsin - Madison, 1550

Engineering Drive, Madison, WI 53706, USA

${ }^{2}$ Medical Physics Department, University of Wisconsin - Madison, 1111

Highland Avenue, Madison, WI 53706, USA

Full list of author information is available at the end of the article
} 
(Continued from previous page)

Conclusions: SHG and optical scattering measurements provide sub-resolution information and when combined provide superior diagnostic power over clinical imaging modalities. Additionally the measurements are able to delineate the different subtypes of ovarian cancer and may potentially assist in treatment protocols. Understanding the altered collagen assembly can supplement histological analysis and provide new insight into the etiology. These methods could become an in vivo screening tool for earlier detection which is important since ovarian malignancies can metastasize while undetectable by current clinical imaging resolution.

Keywords: Ovarian cancer, Second Harmonic Generation (SHG) imaging microscopy, Optical scattering, Extracellular matrix (ECM)

\section{Background}

Ovarian cancer remains the most deadly gynecological cancer among women with an aggregate 5-year survival rate of $\sim 45 \%$. However, the specific rates are highly dependent on the stage of the disease upon diagnosis. For example, diseased localized to the ovary has a 5-year survival rate of $\sim 92 \%$, whereas this decreases precipitously to $27 \%$ for metastatic disease. With current methods only $15 \%$ percent of the patients are diagnosed at stage I (American Cancer Society Cancer Facts and Figure 2015). Early detection is difficult due to vague symptoms (e.g. bloating, abdominal discomfort) as well as the lack of effective clinical screening/imaging tests. Although the CA125 tumor marker and trans vaginal ultrasound (TVUS) have been investigated as screening strategies, both of these methods are not sufficiently selective or specific to be employed as clinical diagnostic tests for early detection of ovarian cancer $[1,2]$. For example, many patients who had frequent screening involving CA-125 blood serum levels and TVUS had already developed widespread high grade ovarian cancer when positively diagnosed [3].

Recently, genetic analysis has led to identification of several subtypes of ovarian tumors [4, 5]. Type I tumors include borderline, mucinous, low-grade serous (LGS), and endometrioid cancers. High-grade serous (HGS) ovarian tumors, which are the predominant type of cancer detected in patients, are classified as Type II tumors. This emerging understanding of classification of ovarian cancer is also leading to the realization that subtype-specific strategies need to be developed for efficient treatment of the malignancy. Therefore, it is not only important for a diagnostic test to detect the presence of ovarian cancer but also to classify the specific subtype of the disease $[6,7]$. No current diagnostic method is able to meet these two criteria.

Examination of remodeling of the extracellular matrix (ECM) through higher resolution and highly specific approaches holds great promise in helping to address these diagnostic needs. This is because these alterations are thought to be a critical step in the initiation and progression of many epithelial carcinomas [8]. These alterations can be in the form of increased collagen content (desmoplasia), modified morphology (e.g. collagen fiber alignment) or up-regulation of different collagen isoforms [9]. Screening techniques that could quantitate changes in the collagen remodeling of the ECM could reveal early changes in architecture which could be used to help detect, identify, and stage disease in patient samples.

To investigate this possibility, we implemented high resolution optical microscopy and spectroscopy tools to quantify changes in the ECM across a spectrum of human ovarian cancers. We use Second Harmonic Generation (SHG) imaging microscopy [10] to objectively quantify differences in ECM structure of normal stromal, type I and II tumors, and also benign lesions. SHG is a coherent process in which two photons are up-converted to exactly twice the frequency (half the wavelength) of an excitation laser. The contrast mechanism of SHG results from a nonlinear polarization given by: $P=\chi^{(2)} E E$ where $\mathrm{P}$ is the induced polarization, $E$ is the electric field vector of the laser, and $\chi^{(2)}$ is the second-order nonlinear susceptibility tensor. The nonlinear susceptibility tensor $\chi^{(2)}$ dictates the intensity of the SHG signal and requires a noncentrosymmetric assembly of harmonophores, which have permanent dipole moments on the size scale of $\lambda_{\mathrm{SHG}}$, to be nonvanishing. This technique is collagen specific and permits imaging deep into tissues (few hundred microns) with intrinsic optical sectioning [11]. The underlying physics permits probing collagen architecture from the macromolecular, supramolecular, and fibril levels through the fiber levels of organization [10].

SHG microscopy has been used in previous studies to investigate the alterations of the stroma in human and mouse models in ovarian cancer using image analysis approaches that interrogated the fiber alignment [12-15]. We previously developed and utilized a more generalizable approach based on the underlying SHG creation physics to differentiate the collagen organization and applied the method to compare HGS tumors and normal stroma [16]. SHG is a coherent process and is dependent on phasematching, $\Delta k=k_{2 \omega}-2 k_{\omega}=0$, (where $\mathrm{k}_{2 \omega}$ and $\mathrm{k}_{\omega}$ are the wave vectors for the SHG and incident photon, respectively). Biological phasematching conditions are not ideal 
(i.e. $\Delta k \neq 0$ ), and as a result of conservation of momentum, there is a specific emission pattern (i.e.forward $\left(\mathrm{F}_{\mathrm{SHG}}\right)$ and backward ( $\left.\mathrm{B}_{\mathrm{SHG}}\right)$ components) that depends on the tissue structure. We thus utilize the intensity ratio, $\mathrm{F}_{\mathrm{SHG}} / \mathrm{B}_{\mathrm{SHG}}$, as a metric that arises from the fibril size and packing relative to the SHG wavelength, $\lambda_{\mathrm{SHG}}$. Phasematching also has implications on the observed SHG intensity, where the relative SHG intensity scales as $\sin (\mathrm{m} \Delta \mathrm{kL} / 2)$ where $m$ is an integer and thus becomes less efficent for larger phase mismatch, i.e. larger $\Delta \mathrm{k}$ values, which correspond to more random structures compared to the length scale of $\lambda_{\mathrm{SHG}}$ [17]. For normal and HGS tumor stroma, we extracted different $\mathrm{F}_{\mathrm{SHG}} / \mathrm{B}_{\mathrm{SHG}}$ values, that based on a mathematical model we developed [17], were consistent with TEM images of the respective collagen fibrils [16]. These differences in structure also led to quantifiable SHG intensity differences between these tissues, where the intensity depends on both the collagen concentration and organization. We further utilized measurements of optical scattering in combination with the SHG metrics. Scattering measurements are also sensitive to ECM architecture and have proven to be highly capable of delineating cancer from normal tissue in many organs $[18,19]$.

We now extend those earlier efforts and perform these measurements and analysis for several tumor types (normal, benign, LGS Type I, endometrioid Type I, and HGS Type II tissues) and also across a large excitation wavelength range (780-1160 $\mathrm{nm})$. We will show the wavelength dependencies encode structural information that identifies changes in collagen fibril/fiber assemblies. All the SHG and optical scattering metrics are reflective of the size and distribution of ECM components relative to the interrogating wavelength. These studies on ECM alterations will lead to better insight into ovarian cancer etiology and progression.

\section{Methods}

\section{Tissue removal and preparation}

Malignant ovarian tissues were obtained using an IRB approved protocol from consented patients undergoing surgical de-bulking treatment for ovarian cancer. Normal ovaries were obtained from consented patients (aged 45-65) undergoing bilateral salpingo-oophorectomy for benign neoplasms, fibroids, endometriosis, and uterine prolapse. All tissues were immediately fixed in $4 \%$ formalin and refrigerated for $24 \mathrm{~h}$ and then switched to phosphate buffered saline (PBS). The specimens were sectioned en face using a Leica Vibratome 1200S (Leica Biosystems, Buffalo Grove, IL) to thickness of $50 \mu \mathrm{m}$ and $100-150 \mu \mathrm{m}$ for optical scattering measurements and SHG imaging studies, respectively. Tissues were classified by a gynecological pathologist into normal $(n=4)$, benign $(n=4)$, borderline/low-grade serous Type I $(n=4)$, endometrioid Type I $(n=3)$, and high-grade serous Type II $(n=3)$. The genomic mutations of the tissues are not known.

\section{SHG microscopy \\ SHG imaging system}

The essentials of the SHG imaging system has been described elsewhere [10]. A femtosecond laser was coupled to a home-built laser-scanning system (WiscScan; http:// loci.wisc.edu/software/wiscscan) on a fixed stage upright microscope base (BX61WI, Olympus, Center Valley, PA) microscope. A $40 \times 0.8$ numerical aperture $(\mathrm{NA})$ water immersion objective and a 0.9 NA condenser are used for excitation and collection, respectively, of the forward SHG signal providing lateral and axial resolutions of approximately 0.7 and $2.5 \mu \mathrm{m}$, respectively. The backward SHG was collected in a non-descanned geometry, where the detector was in the infinity space. Both detectors are H7422-40P GaAsP photomultiplier tubes (Hamamatsu, Hamamatsu, Japan). Calibration of the forward and backward detection pathways was performed using the two-photon excited fluorescence imaging of beads emitting in the same wavelength range as the collected SHG signal. The laser excitation range was $780-1160 \mathrm{~nm}$ provided by Chameleon Ultra Ti:Sapphire oscillator and a synchronously pumped APE Optical Parametric Oscillator (Coherent, Santa Clara, CA). For all excitations, the SHG signal was isolated with $20 \mathrm{~nm}$ full width half maximum bandpass filters centered at the corresponding SHG wavelength (Semrock, Rochester, NY). Circularly polarized light, determined at the focus, was used throughout to equally excite all fiber orientations [10].

\section{D SHG imaging}

Depth dependent Forward/Backward (F/B) measurements were made for the entire thickness of the ovarian sections in three different locations, from which the data was obtained sequentially across the wavelength range. The SHG intensities were integrated across the whole fields of view using both FIJI (an open-source Image) platform for image analysis) [20] and MATLAB (MathWorks, Natick, MA). The measured forward attenuation, i.e. the rate of SHG intensity decrease with increasing depth into tissue, was also used in characterizing tissue alterations. Due to intrinsic heterogeneity in concentration of biological tissues, we found it necessary to normalize the SHG intensity response to account for local variability within the same tissue (different fields of view) and to make relative comparisons between tissues. Normalization of each optical section within each optical series was selfnormalized with the average maximum intensity value. The normalized forward attenuation and the F/B data were taken concurrently. 3D renderings were performed in Imaris (Bitplane AG, Zurich, Switzerland). 


\section{Monte Carlo simulations}

The SHG directional emission ratio, $\mathrm{F}_{\mathrm{SHG}} / \mathrm{B}_{\mathrm{SHG}}$, and relative SHG conversion efficiency were decoupled from the depth dependent SHG response curves using Monte Carlo simulations based on an adapted MCML (Monte Carlo Multi-Layer) [21] framework [22, 23]. The $\mathrm{F}_{\mathrm{SHG}} /$ $\mathrm{B}_{\mathrm{SHG}}$ at each wavelength was extracted by running a series of forward simulations of the measured forward/ backward vs depth curve based on the corresponding measured optical properties (described below) and initial guesses of the emission directionality and then obtaining the best fit to the simulations. The relative SHG conversion efficiency at each excitation wavelength was determined by modeling the normalized forward SHG attenuation response using a similar simulation incorporating the extracted $\mathrm{F}_{\mathrm{SHG}} / \mathrm{B}_{\mathrm{SHG}}$ and wavelength specific optical parameters at both the excitation and SHG wavelengths. All forward simulations were completed using parallel computing at the Wisconsin Center for High Throughput Computing at the University of Wisconsin-Madison and the values were stored in tables, permitting all the image processing to be performed in custom MATLAB scripts.

\section{Optical scattering measurements}

We determined the optical properties of tissue through an average of three independent locations within a tissue specimen, where these parameters include the refractive index $n$, absorption coefficient $\mu_{a}$, scattering coefficient $\mu_{s}$, and scattering anisotropy $g$. An estimate of the bulk refractive index was determined by a total internal reflection measurement of the sample as described by $\mathrm{Li}$ et al. [24]. The absorption coefficient is negligible in most fibrillar tissues as $\mu_{a}<<\mu_{s}$ and was previously confirmed in ovarian tissues [16].

The scattering coefficient and anisotropy were obtained independently in a multi-step process using onaxis attenuation measurements which is applicable when $\mu_{a}<<\mu_{s}$ [25]. These values were measured at 390, 445, $494,535,780,890,988$, and $1070 \mathrm{~nm}$ using the tunable Ti: Sapphire laser tunable and an external BBO frequency doubling crystal. SHG imaging was used to determine the tissue thickness to obtain $\mu_{s}$ from the Beer-Lambert law. We then report the reduced scattering coefficient, $\mu_{s}{ }^{\prime}$, which is a merged property defined by:

$$
\mu_{s}^{\prime}=\mu_{s}(1-g)
$$

\section{Statistical analysis}

The canonical variable linear discriminant and logistic progression were performed in SAS (SAS Institute Inc., Chicago, IL) using the CANDISC procedure, where this was applied to all sample data points. Using the canonical variable weights, a model based on the relative SHG conversion efficiency, $\mathrm{F}_{\mathrm{SHG}} / \mathrm{B}_{\mathrm{SHG}}, \mu_{s}$, and their wavelength dependence was used to classify the images. ROC curves were computed by comparing the modeled response versus the true or assigned tissue classification via a logistic regression. All the ANOVA and Fisher LSD statistical tests were performed in Origin 9.1 (OriginLab, Northampton, $\mathrm{MA})$. For the latter, $p<0.05$ was considered significant.

\section{Results \\ Fiber morphology}

The left and right columns of Fig. 1 show a representative 3D SHG rendering and H\&E histology image of each tissue type studied in this paper. Normal post-menopausal ovarian tissues have a loose-mesh like collagen network, where the "holes" in the images correspond to stromal fibroblasts, which are transparent in SHG contrast. Malignant tissues are heterogeneous in nature; however, HGS tissue morphology is highly conserved within the patient population displaying a dense, highly aligned network of long wavy collagen fibers. LGS tissues are fibrotic with tightly packed shorter collagen fibers whereas endometrioid tissues have low collagen density and highly aligned thin collagen fibers. Benign tissues are fibrotic with wavy networks of large collagen fibers. We note that there is not significant variation in the collagen morphology across the different sampling regions as we limit the analysis $\mathrm{s}$ to collage rich areas near the surface epithelium. These overall patterns in each case are also observed in the corresponding H\&E sections visualizing the collagen and cell nuclei.

It is difficult to precisely determine fiber diameters as they are not all discrete and often overlap within the resolution of the microscope. However, we approximated the diameters by thresholding and measuring the thickness by creating a profile across several discretized fibers. We then found average values (standard error in parenthesis) of: normal: $2.7(0.1) \mu \mathrm{m}$; benign: $3.6(0.2)$ $\mu \mathrm{m}$; endometrioid: $1.9(0.1) \mu \mathrm{m}$; LGS: $3.4(0.2) \mu \mathrm{m}$ and HGS: $2.3(0.1) \mu \mathrm{m}$. The diameters of the LGS and benign were similar; those of the endometrioid and HGS were similar, but all the others were different from each other.

\section{Tissue scattering properties}

The wavelength dependence of the reduced scattering coefficient, $\mu_{s}{ }^{\prime}$, depends on the spatial distribution of the refractive index due to structural differences on size scales smaller than the diffraction limit. For rigorous tissue characterization, Backman et al. used the flexible Whittle-Matérn correlation function to quantify the distribution of length scales where the output is the shape factor $\mathrm{m}$, which corresponds to one half of the fractal dimension [26, 27]. The shape factor is connected to the spectral dependence of the reduced scattering coefficient 


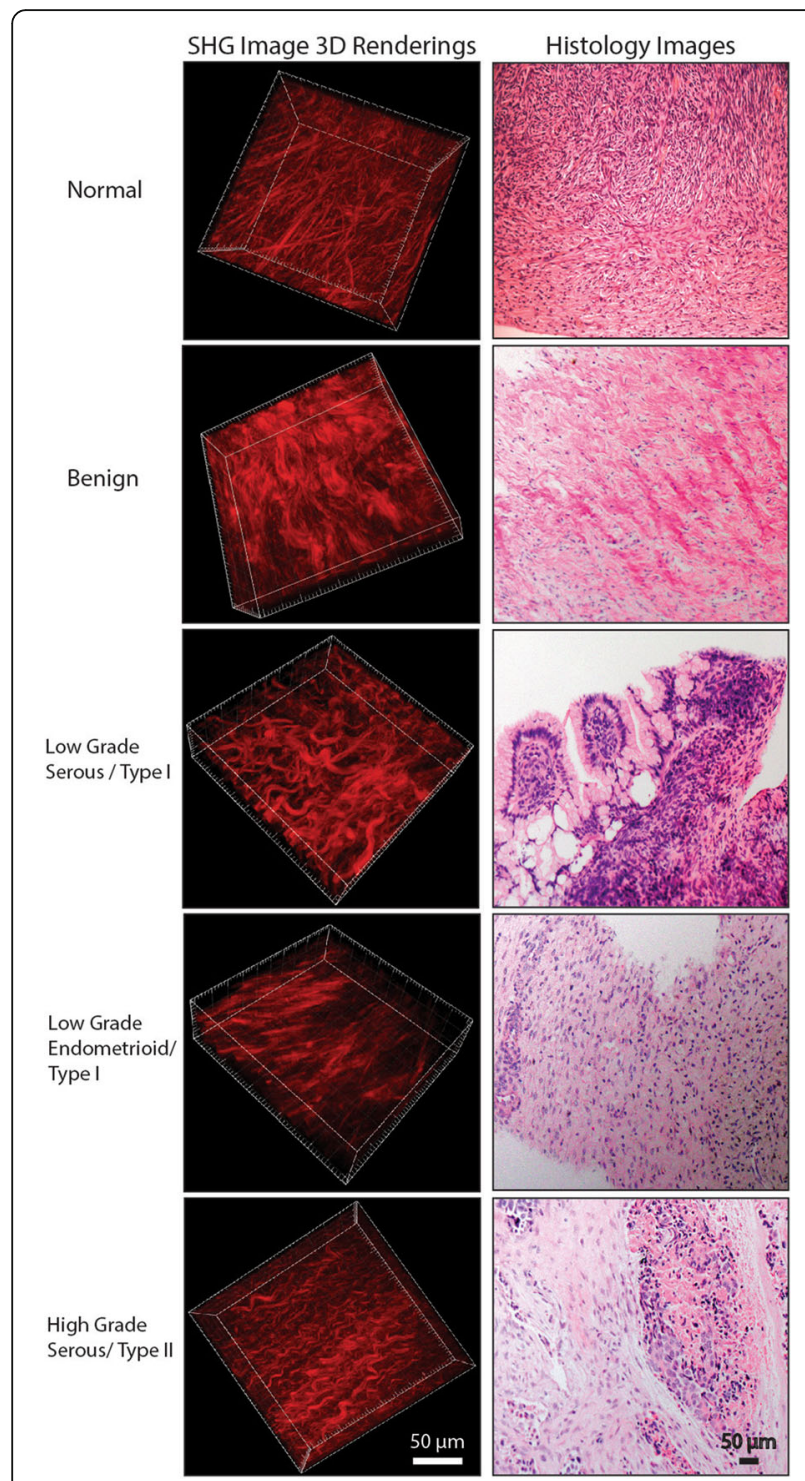

Fig. 1 Left column shows 3D renderings of forward directed SHG images of representative normal stroma, benign, LGS, endometrioid, and HGS ovarian tumors obtained at $890 \mathrm{~nm}$ excitation. The tissue sections were $\sim 100 \mu \mathrm{m}$ in thickness. Right column is representative H\&E staining of the same tissue. Scale bar $=50 \mu \mathrm{m}$

through the power law expression by: $\mu_{\mathrm{s}}^{\prime}(\lambda) \sim \lambda^{(2 \mathrm{~m}-4)}$ where higher $m$ values are associated with larger, more ordered structures on the approximate size scale of $50 \mathrm{~nm}$ to $1 \mu \mathrm{m}$.

We measured the spectral dependence of the reduced scattering coefficient, $\mu_{s}{ }^{\prime}$, for the five tissue types over the range of 390-535 $\mathrm{nm}$ (corresponding to the wavelengths where the SHG metrics were probed) and the results are shown in Fig. 2. The spectral dependencies of the normal and malignant ovarian tissues are different. HGS ECM had the greatest scattering intensity, indicating increased tissue density. This is consistent with the

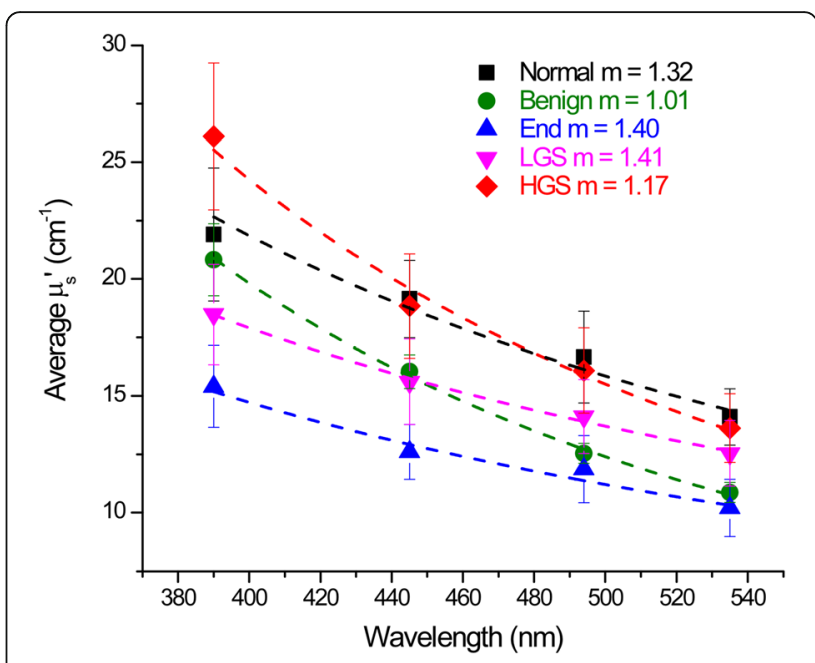

Fig. 2 Wavelength dependence of the reduced scattering coefficient $\mu_{s}{ }^{\prime}$ over the wavelength range used for SHG imaging (390-535 nm) for the normal stroma and ovarian tumors. The best fit to the scattering power law from the corresponding $m$ factor is shown with the experimental data. All curves are the average response from normal $n=4$; benign $n=4$; endometrioid $n=3$; LGS $n=4$; and $\operatorname{HGS} n=3$. Error bars are standard error

dense fibrillar structure seen in the $3 \mathrm{D}$ rendering in Fig. 1. The endometrioid tumors had the weakest absolute scattering, which is also consistent with the SHG images showing sparse (but regular) fibers. We stress that the measured $\mu_{s}^{\prime}$ values reflect both the fiber and cellular components, however the former is much more strongly scattering. The other tissues (LGS, normal and benign) showed intermediate behavior. These were mostly statistically different at $390 \mathrm{~nm}(p<0.05)$, and some were significant at other wavelengths. In addition to the absolute scattering intensities, we also compared the tissues through the fitted $m$ values. By this metric, LGS and endometrioid tissues are the most ordered with $m$ values of 1.41 and 1.40, respectively, where these were not distinct; however, the $\mu_{s}{ }^{\prime}$ were different at 390 and $445 \mathrm{~nm}$. Benign tissues $(m=1.01)$ show the least order, followed by HGS $(m=1.17)$, then normal tissues $(m=1.32)$. Given that both $\mu_{s}{ }^{\prime}$ and $m$ are different for some of the tissues, we concluded that both the scattering intensity and spectral dependence are needed to describe the tissues fully and provide the best differentiation. We note that this is not unexpected as the $m$ values correspond to organization on the $\sim 50 \mathrm{~nm}-1 \mu \mathrm{m}$ size scale, while $\mu_{s}{ }^{\prime}$ is a merged parameter reflecting both organization $(\mathrm{g})$ and density $\left(\mu_{s}\right)$. Thus, higher values of both parameters may not occur for a single tissue.

\section{Wavelength dependence of $\mathrm{F}_{\mathrm{SHG}} / \mathrm{B}_{\mathrm{SHG}}$}

The $\mathrm{F}_{\mathrm{SHG}} / \mathrm{B}_{\mathrm{SHG}}$ metric is a subresolution parameter that arises from the fibril size and packing relative to $\lambda_{\mathrm{SHG}}$ [17]. The wavelength dependence of this parameter can 
yield further discrimination than possible by single wavelength measurements as tissues having differing fibril architecture relative to the SHG wavelengths will have a different wavelength dependent $\mathrm{F}_{\mathrm{SHG}} / \mathrm{B}_{\mathrm{SHG}}$. Here we utilize a broad excitation range $(780-1160 \mathrm{~nm})$, which is limited by optical transmission of the SHG $(390 \mathrm{~nm})$ and excitation $(1160 \mathrm{~nm})$ wavelengths and then extract $\mathrm{F}_{\mathrm{SHG}} / \mathrm{B}_{\mathrm{SHG}}$ as described in the Methods section of this manuscript.

The data are summarized in Fig. 3a. The optimized simulation in each case was not statistically different (via $X^{(2)}$ test) from the measured data, implying a good fit to $\mathrm{F}_{\mathrm{SHG}} / \mathrm{B}_{\mathrm{SHG}}$. All ovarian tissue types displayed an increase in $\mathrm{F}_{\mathrm{SHG}} / \mathrm{B}_{\mathrm{SHG}}$ emission directionality with increasing wavelength. This result is expected due to phasematching considerations, as we have reported for other tissues [23]. This arises because the dispersion in refractive index between the laser excitation and SHG wavelengths decreases at longer wavelengths, decreasing the phase mismatch efficiency and increasing the forward SHG component [17].

However, the form of the wavelength dependent increase in $\mathrm{F}_{\mathrm{SHG}} / \mathrm{B}_{\mathrm{SHG}}$ is tissue specific. HGS tumors have the smallest $\mathrm{F}_{\mathrm{SHG}} / \mathrm{B}_{\mathrm{SHG}}$. One physical scenario that gives rise to a low $\mathrm{F}_{\mathrm{SHG}} / \mathrm{B}_{\mathrm{SHG}}$ is when the fibril size and spacing are regular and much smaller than $\lambda_{\mathrm{SHG}}$ where this results in efficient quasi-phasematching (QPM), thus increasing the $\mathrm{B}_{\mathrm{SHG}}$ emission $[17,28]$. We previously verified this through TEM imaging of HGS tumors [16]. The higher emission directionality for the LGS ECM implies that the collagen fibrils are larger and more disorganized than those in both normal and HGS tissues. Overall, benign serous tissues have the highest $\mathrm{F}_{\mathrm{SHG}} / \mathrm{B}_{\mathrm{SHG}}$ emission directionality indicating this tissue type has the largest collagen fibrils. This is also suggested by this tissue having a

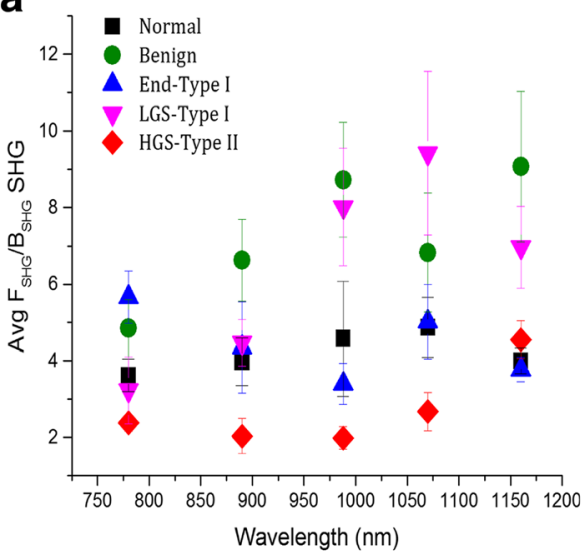

C

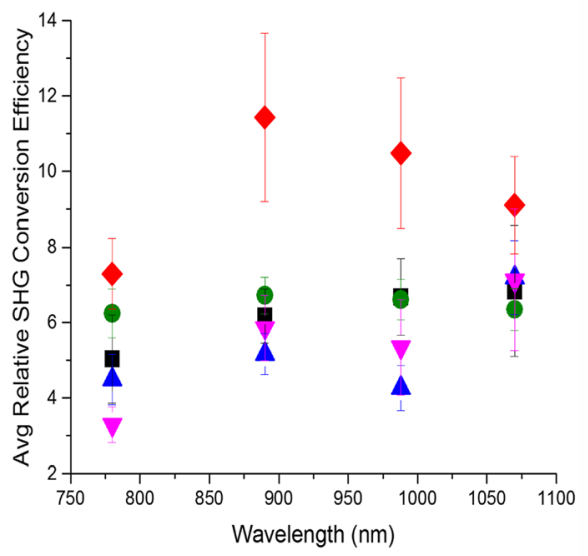

b

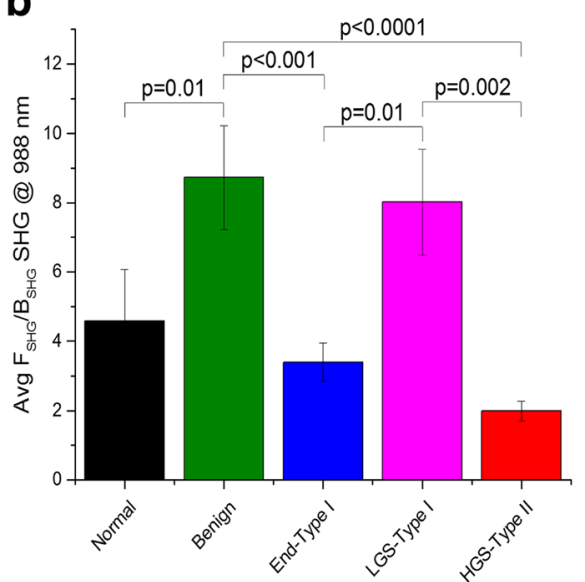

d

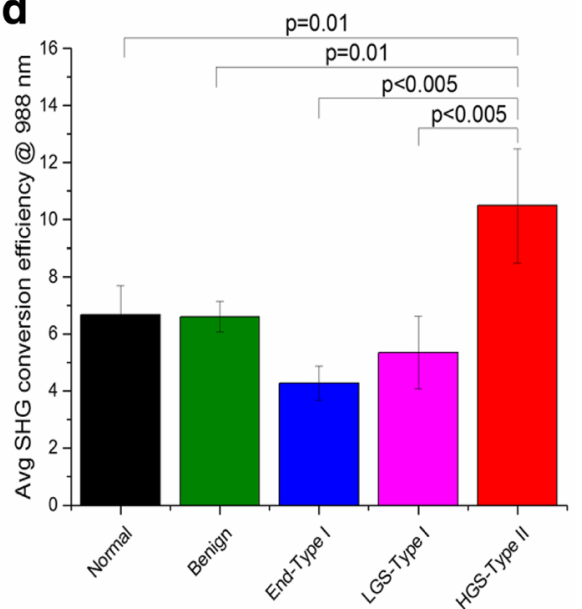

Fig. 3 Extracted $\mathrm{F}_{S H G} / B_{S H G}$ emission directionality and SHG conversion efficiencies of the ovarian tissues obtained via Monte Carlo simulations. a Wavelength dependent $F_{S H G} / B_{S H G}$ emission directionality response from 780 to $1160 \mathrm{~nm}$ (excitation wavelengths) and $\mathbf{b}$ Average $F_{S H G} / B_{S H G}$ emission directionality at $988 \mathrm{~nm}$, where best delineation between the tissues was obtained; $\mathrm{p}$ values showing significant differences are indicated. c Wavelength dependent SHG conversion efficiency response from 780 to $1070 \mathrm{~nm}$ excitation wavelengths. d Relative conversion efficiencies at $988 \mathrm{~nm}$, where best delineation between tissues was obtained; $\mathrm{p}$ values showing significant differences are indicated. All curves are the average response from normal $n=4$; benign $n=4$, endometrioid $n=3$; LGS $n=4$; and $\operatorname{HGS} n=3$. Error bars are standard error 
the largest measured fibers from Fig. 1. Normal tissues have intermediate $\mathrm{F}_{\mathrm{SHG}} / \mathrm{B}_{\mathrm{SHG}}$ values between $\mathrm{HGS}$ and LGS tissues, demonstrating that both these tissues have altered collagen assembly than normal post-menopausal ovarian stroma. However, the alterations result in opposite trends, suggesting the modifications have different forms. This is consistent with type I and type II classes representing genetically different diseases [4].

The optimal single wavelength to differentiate the tissue types by the $\mathrm{F}_{\mathrm{SHG}} / \mathrm{B}_{\mathrm{SHG}}$ metric was $988 \mathrm{~nm}$. The $\mathrm{F}_{\mathrm{SHG}} /$ $\mathrm{B}_{\mathrm{SHG}}$ values and statistical analysis at this wavelength are shown in Fig. 3 b. The $\mathrm{F}_{\mathrm{SHG}} / \mathrm{B}_{\mathrm{SHG}}$ statistically differentiates the collagen fibril size and assembly of HGS tissues from LGS tissues and also from benign tissues $(p \leq 0.002)$ despite small samples sizes of 3 or 4 patients per tissue type. Benign tissues are also statistically different $(p \leq 0.01)$ from normal and endometrioid tissues. Also interesting is the difference in collagen fibril assembly of LGS and the endometrioid tissues $(p=0.01)$ highlighting the heterogeneity of collagen features within these tissues, even though both are classified as Type I [5]. Although $988 \mathrm{~nm}$ excitation, provided statistical distinction in the collagen fibril assembly between different types of ovarian cancer, this metric by itself was not able to provide necessary separation between normal and LGS and normal from HGS tumors. However, these were differentiated by including this metric in the multivariate analysis to be shown later.

\section{SHG conversion efficiency}

We also determined the wavelength dependence of the relative SHG conversion efficiency as a means to characterize the tissue structure. The conversion efficiency is a coupled effect of the collagen concentration (square thereof) and its fibrillar organization on the size scale of $\lambda_{\text {SHG }}[16,22]$. Monte Carlo simulations using the measured optical properties at the fundamental and SHG wavelengths extracted the SHG conversion efficiency by obtaining the best fit to the conversion efficiency, in analogy with obtaining the $\mathrm{F}_{\mathrm{SHG}} / \mathrm{B}_{\mathrm{SHG}}$ values.

The extracted SHG conversion efficiencies for the five tissue types across the 780-1070 $\mathrm{nm}$ range are shown in Fig. 3c. As absolute values of the conversion efficiency are not readily obtainable, we plot them normalized to the maximum intensity (here HGS at $890 \mathrm{~nm}$ ). Overall, the HGS tissues have the most efficient SHG relative to the normal and other ovarian cancer subtypes, indicating the highest collagen concentration and/or organization. We note that these properties are not separable by SHG imaging. Optimal values of relative SHG conversion efficiency were found at $988 \mathrm{~nm}$. Here, the HGS tissues were statistically different $(p \leq 0.01)$ from normal and all other types of ovarian cancer. LGS, endometrioid, benign, and normal tissues had similar values of relative SHG conversion efficiency; however, the wavelength dependent response is slightly different. LGS tissues have the greatest wavelength dependent increase in the SHG conversion. We note that the HGS conversion efficiency decreases with wavelength, whereas the other tissues show an increase, suggesting large structural differences which will be addressed in the Discussion.

\section{Multi-variable linear discriminant analysis}

In this study, three sub-optical resolution properties were obtained to further understand the ECM remodeling associated with ovarian cancer. Combining these independent metrics provides a more robust characterization of the normal and diseased ovarian stroma. This is important as not all parameters at all wavelengths were statistically different. To this end, we used a linear discriminant with three canonical variables (see methods), where the results are summarized in Table 1. All the individual cells in Table 1 are pairwise comparisons between the tissue types, as opposed to one versus the rest classification. These metrics successfully classified HGS from normal, benign, LGS, and endometrioid tissues with excellent accuracy indicating that this multi-variable approach has high fidelity in describing the altered ECM architecture. Normal stroma was moderately differentiated from benign, LGS, and endometrioid with accuracies between 75 and $80 \%$. Even this moderate differentiation is comparable to the collective diagnostic accuracy of clinical imaging modalities. LGS and benign tumors had the poorest differentiation ( $65 \%)$ which is expected as these tissues had the most similar morphology (Fig. 1) [29].

\section{Discussion}

We previously developed a heuristic model based on phasematching between the excitation and SHG waves that correlates collagen fibril size and assembly with the $\mathrm{F}_{\mathrm{SHG}} / \mathrm{B}_{\mathrm{SHG}}$ emission directionality [17]. In this context, we defined a "domain," meaning either a single fibril/ fiber or smaller fibrils overlapped together to achieve the

Table 1 Classification accuracy of ovarian tissues based on the multi-parameter canonical linear discriminant model

\begin{tabular}{lllll}
\hline & High-grade serous $(n=3)$ & Benign $(n=4)$ & Low-grade serous $(n=4)$ & Endometrioid $(n=3)$ \\
\hline Normal $(n=4)$ & $89.6 \%$ & $77.6 \%$ & $77.8 \%$ & $79.3 \%$ \\
Benign $(n=4)$ & $93.7 \%$ & & & \\
Endometrioid $(n=3)$ & $95.6 \%$ & $76.6 \%$ & $69.7 \%$ & \\
Low-grade serous $(n=4)$ & $96.9 \%$ & $67.3 \%$ & & \\
\hline
\end{tabular}


same diameter. We showed that larger domains on the size scale of $\lambda_{\mathrm{SHG}}$ produce larger $\mathrm{F}_{\mathrm{SHG}} / \mathrm{B}_{\mathrm{SHG}}$, where smaller sizes yield lower values, and also weaker SHG intensities. A special case can arise, when the fibrils are much smaller than $\lambda_{\mathrm{SHG}}$ and equally spaced, through QPM, producing relatively more efficient backward SHG.

The phasematching arising from the distribution of fibril sizes is always relative to $\lambda_{\mathrm{SHG}}$; thus, if the tissues have different distributions of domain sizes, this will result in a different wavelength dependence of the $\mathrm{F}_{\mathrm{SHG}} /$ $\mathrm{B}_{\mathrm{SHG}}$ parameter. The overall increase in $\mathrm{F}_{\mathrm{SHG}} / \mathrm{B}_{\mathrm{SHG}}$ with increasing wavelength is expected by simple phasematching conditions based on the wavelength dependence of the refractive index [23]. The other factor of this increase is the extent the domain matches the size scale of $\lambda_{\text {SHG. }}$ The HGS tissues show the weakest wavelength dependence, and this is consistent with our previously reported TEM images, which showed the fibrils were all $\sim 60 \mathrm{~nm}$ in diameter [16]. Physically this would correspond to near single value of the phase mismatch and should result in little wavelength dependence as the fibrillar domain is also much smaller than $\lambda_{\mathrm{SHG}}$. The low $\mathrm{F}_{\mathrm{SHG}} /$ $\mathrm{B}_{\mathrm{SHG}}$ is further consistent with arising from the QPM mechanism. While we do not have TEM images for the LGS and benign tissues, within the resolution of the microscope (Fig. 1), these fibers are larger than the HGS and normal stroma and have larger $\mathrm{F}_{\mathrm{SHG}} / \mathrm{B}_{\mathrm{SHG}}$ values and have a more pronounced wavelength dependence. The large wavelength dependent increase was observed for the LGS tumors, indicating a broader distribution of fibril sizes. Similar to the HGS tumors, the endometrioid tumors have a weak wavelength dependence of $\mathrm{F}_{\mathrm{SHG}} / \mathrm{B}_{\mathrm{SHG}}$ values and also narrow distribution of fibers sizes as seen in Fig. 1. In sum, this analysis determining the wavelength dependence of $\mathrm{F}_{\mathrm{SHG}} / \mathrm{B}_{\mathrm{SHG}}$ values affords obtaining sub-resolution structural information of the fibril size and packing in intact tissues without performing TEM analysis as seen in Fig. 4.

Differences in collagen density and organization are also manifested in the relative SHG conversion efficiencies. The SHG conversion efficiency is based both on the magnitude of nonlinear susceptibility tensor $\chi^{(2)}$ which is a property of the collagen itself, and phasematching, which arises from the organization relative to $\lambda_{\mathrm{SHG}}$ [17]. We recently reported the wavelength dependence of the SHG in murine tendon, and showed a sharp decrease in conversion efficiency ( $\sim 5$ fold) over the same wavelength range used here. This decrease was ascribed mostly to decreasing $X^{(2)}$ rather than the improved phasematching at longer wavelengths [30]. Furthermore, this was physically reasonable as the diameters of murine tendon fibrils are narrowly distributed [31], and as discussed above, this leads to a weak wavelength dependence of phasematching as well as a small change in the concomitant $\mathrm{F}_{\mathrm{SHG}} / \mathrm{B}_{\mathrm{SHG}}$ values. Here, we performed this analysis on the different ovarian ECM tissues and compared their relative conversion efficiencies and wavelength dependence.

As shown in Fig. 3c, the HGS tissues had the highest SHG conversion efficiency. This result was expected based on the appearance of the collagen morphology in Fig. 1 and strongest scattering (due to highest density) in Fig. 2. We also point out that the HGS tissues had the opposite dependence on wavelength, where the relative conversion efficiency decreased instead of the increase with longer wavelengths seen from the other tissues. The HGS response is quite similar to that observed for tendon in our prior work [30], where the $\chi^{(2)}$ component dominates the increased phasematching at longer wavelengths. This is further consistent with the lower $\mathrm{F}_{\mathrm{SHG}} /$
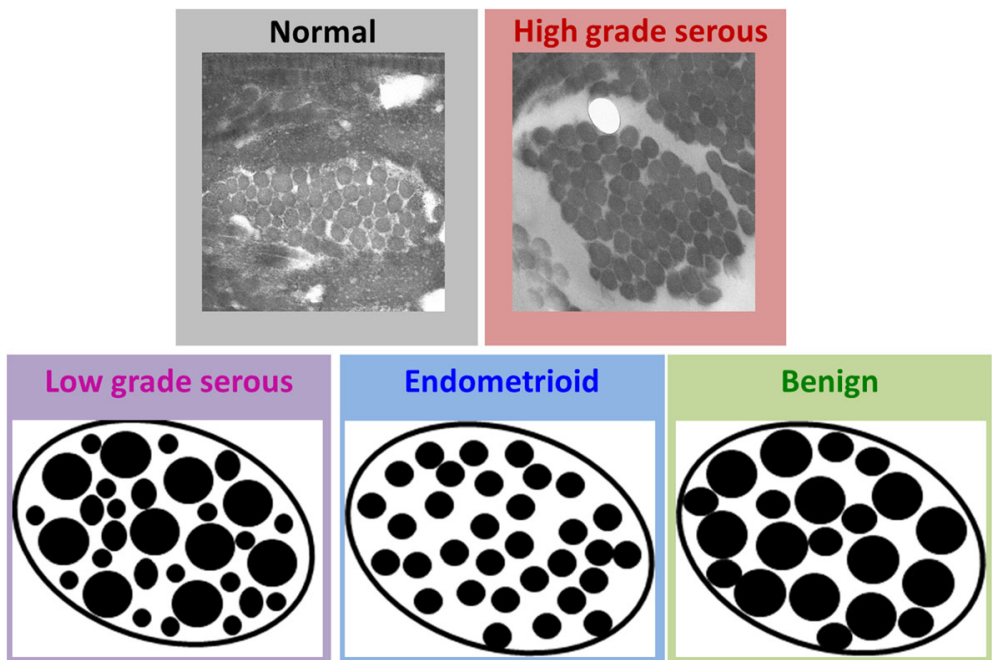

Fig. 4 Collagen fibril assembly based on the wavelength dependent phasematching response. TEM images of normal and HGS ovarian tissues. Cartoons of the LGS, endometrioid, and benign ovarian tissues 
$\mathrm{B}_{\mathrm{SHG}}$ emission directionality shown in Fig. 3a and implies the fibril structure is small relative to $\lambda_{S H G}$ with a narrow distribution of diameters. The relative SHG conversion efficiencies were similar at $988 \mathrm{~nm}$ (like the $\mathrm{F}_{\mathrm{SHG}} / \mathrm{B}_{\mathrm{SHG}}$ values). All the other tissue types showed a slight increase with wavelength. From a structural perspective, this implies the fibril domain is larger than the HGS tissues and is also characterized by a larger distribution of fibril sizes (Fig. 4). Thus, while arising from different attributes, the emission directionality and relative conversion efficiencies both yield consistent structural information. Importantly, these structural aspects arise from features below the resolution limit of the microscope but are obtainable due to the physical underpinnings of the SHG process.

We also characterized the tissue architecture using optical scattering measurements of both the scattering intensity and its wavelength dependence (Fig. 2) $[18,19,26]$. The $\mu_{s}{ }^{\prime}$ and fit $m$ values were obtained over the SHG wavelength range and good separation was achieved between most of the tissue types. We also performed this fit across the entire spectral range that included all the excitation and SHG wavelengths used in the SHG analyses. This approach yielded almost no discrimination between the tissues, where the reduced scattering coefficients at the longer wavelengths $(>780 \mathrm{~nm})$ were largely similar. In analogy to the SHG data, the scattering coefficients are also related to the scatterer size relative to the interrogating wavelength. The higher sensitivity at the shorter wavelengths implies that the scattering structures lie within this size range. Although optical scattering and SHG arise from different physics, we found similar size scales are operative in both cases. The data identified the most sensitive wavelength range to use for diagnostic purposes for both SHG and optical scattering.

Although the individual metrics, $\mathrm{F}_{\mathrm{SHG}} / \mathrm{B}_{\mathrm{SHG}}$ emission directionality, relative SHG conversion efficiency, $\mu_{s}^{\prime}$, and their respective wavelength dependence provided differentiation in some cases, each metric described only a single feature of the ovarian ECM. Combination of the metrics in a canonical linear discriminant provided enhanced classification based on the weights of the three individual variables.
Robust classification (>90\%) of high grade serous from all other ovarian tissue types was achieved. This is arguably the most important task as these tumors account for $70 \%$ of all ovarian malignancies and have the poorest survival rates. Moderately accurate classification was obtained for normal tissues relative to the other types. Interestingly, the least accuracy was obtained for LGS and benign tumors (Table 1). It has been suggested that the latter can evolve into LGS [5]; thus, if this supposition is correct, this poor discrimination would be expected.

Collectively the SHG and scattering metrics provided structural analyses of different aspects of structure for the different tissue types. These aspects are summarized in Table 2, where the readouts for classification are compared relative to the HGS tissues. For example, if only $\mu_{s}$ values, which are primarily a measure of tissue density, were used to classify the tissues, both normal and HGS tissues were similar. However, with the inclusion of the $m$ fit to the wavelength dependence, the $\mathrm{F}_{\mathrm{SHG}} / \mathrm{B}_{\mathrm{SHG}}$ emission directionality, and the relative SHG conversion efficiency, we note that the normal and HGS are discriminated, indicating they have very different ECM structural aspects. Using all these metrics and their structural meanings, not only greatly enhanced the classification of the tissues, but also helped to understand the importance of different physical features within the tumor microenvironment.

In principle, all the metrics used here could be performed in conjunction with a laparoscope in a minimally invasive manner. For example, there already has been one report of microendoscopy for ovarian cancer [15]. In principle, such a design could be adapted to probe the SHG directionality (our work in progress). Currently, all women with suspected masses undergo oophorectomies even though these masses are frequently pathologically benign. However, SHG/optical scattering interrogation could potentially spare the ovaries if tumors were deemed to be benign. Moreover, the treatment course could be different if LGS versus HGS disease is found. More importantly, SHG/optical scattering could be used as a screening modality for early diagnosis of Type I and Type II ovarian

Table 2 Summary of independent metrics and their physical meaning relative to high-grade serous - Type II tissues

\begin{tabular}{|c|c|c|c|c|}
\hline & $\overline{\mu_{s}^{\prime}}$ & m shape value & $\mathrm{F}_{\mathrm{SHG}} / \mathrm{B}_{\mathrm{SHG}}$ & Conversion efficiency \\
\hline Normal & $\begin{array}{l}(\approx) \\
\text { Density }\end{array}$ & $\begin{array}{l}1.32(++) \\
\text { Cell and collagen structures more ordered }\end{array}$ & $\stackrel{+}{\text { Disordered similar sized collagen fibrils }}$ & $\begin{array}{l}(--) \\
\text { Less collagen organization } \\
\text { at fiber level }\end{array}$ \\
\hline Benign & $\begin{array}{l}(-) \\
\text { Density }\end{array}$ & $\begin{array}{l}1.01(---) \\
\text { Cell and collagen structures less ordered }\end{array}$ & $\begin{array}{l}(++) \\
\text { Large collagen fibrils }\end{array}$ & $\begin{array}{l}(-) \\
\text { Less collagen organization } \\
\text { at fiber level }\end{array}$ \\
\hline Endometrioid - Type I & $\begin{array}{l}(--) \\
\text { Density }\end{array}$ & $\begin{array}{l}1.40(+++) \\
\text { Cell and collagen structure more ordered }\end{array}$ & $\begin{array}{l}(+) \\
\text { Disordered similar sized collagen fibrils }\end{array}$ & $\begin{array}{l}(-) \\
\text { Decrease collagen density }\end{array}$ \\
\hline Low-grade serous - Type I & $\begin{array}{l}(-) \\
\text { Density }\end{array}$ & $\begin{array}{l}1.41(+++) \\
\text { Cell and collagen structure more ordered }\end{array}$ & $\begin{array}{l}(++) \\
\text { Larger more disordered collagen fibrils }\end{array}$ & $\begin{array}{l}(-) \\
\text { Less collagen organization } \\
\text { at fiber level }\end{array}$ \\
\hline
\end{tabular}

(+) slightly increased, (++) moderately increased, (+++) highly increased, $(\approx)$ similar valued, $(-)$ slightly decreased, (- -) moderately decreased, (- - -) highly decreased 
cancer in postmenopausal women or high risk subjects (up to 30 fold) with a family history of this disease or known BRCA mutations.

\section{Conclusions}

We have shown that SHG imaging microscopy and optical scattering measurements characterize the underlying ECM alterations in ovarian cancers and benign lesions relative to normal stroma. These approaches interrogate structures below the resolution of microscopy and provide collagen specific readouts. These quantitative methods are especially powerful when examining the wavelength dependencies as different tumor types have differing architectures relative to the interrogating spectrum. The ability to distinguish different classifications of ovarian cancer based on the collagen ECM is important for several reasons including: i) increased accuracy of clinical classification without the need of genomic analysis; ii) improve our understanding of the different etiologies of different ovarian cancers; and iii) potential to develop ovarian cancer type specific chemotherapeutics. This work provides the core data to show the potential of ECM collagen measurements as a new image-based biomarker for ovarian cancer. Future efforts will include further examination of collagen associated metrics, clinical trials to further show the linkage between collagen changes and clinical outcome, and the design of instrumentation that can exploit this information for clinical use.

\section{Abbreviations \\ ECM: Extracellular matrix; $F_{S H G} / B_{S H G}$ : $S H G$ directional emission ratio; g: Tissue anisotropy; HGS: High Grade Serous; LGS: Low Grade Serous: QPM: Quasiphasematching; SHG: Second Harmonic Generation; TEM: Transmission electron microscopy; $\lambda_{S H G}$ : SHG wavelength; $\mu_{s}^{\prime}$ : Reduced scattering coefficient; $X^{(2)}$ : Nonlinear susceptibility tensor}

\section{Acknowledgements}

We would like to thank Peter Crump for his assistance and guidance with the statistical analysis.

\section{Funding}

This work was supported by the National Science Foundation under CBET 1402757, CBET-0959525 and by the National Institute of Health NIH 5T32CA009206-34.

\section{Availability of data and materials}

Image data is available upon request.

\section{Authors' contributions}

KBT prepared the specimens, acquired, processed and analyzed the SHG imaging data, and assisted in drafting the manuscript. KRC performed all the optical scattering measurements, Monte Carlo simulations, and assisted in drafting the manuscript. KWE worked on the instrument design. MP co-conceived the project and assisted in acquiring tissue specimens. SMS provided ovarian cancer insight and acquired tissues. PJC co-conceived the project and assisted in drafting the manuscript. All authors read and approved the final manuscript.

\section{Authors' information}

KBT received her Ph.D in biomedical engineering from the University of Wisconsin-Madison and is currently an assistant professor at the University of Maine-Orono. She is a member of Optical Society of America (OSA). KRC is a Ph.D student in biomedical engineering at the University of WisconsinMadison and is a member of both Optical Society of America (OSA) and the International Society for Optics and Photonics (SPIE). KWE received his Ph.D from the University of Wisconsin-Madison and is the Director and Principal Investigator at the Laboratory for Optical and Computational Instrumentation (LOCl) at the University of Wisconsin-Madison. MP received his Ph.D from Old Dominion University and Eastern Virginia Medical School in biomedical sciences and currently is an associate professor in the Department of Obstetrics and Gynecology at the University of Wisconsin-Madison. SMS received her M.D. from University of Khartoum School of Medicine in Sudan and is currently an associate professor in the Department of Obstretrics and Gynecology at West Virginia University. PJC received his Ph.D in physical chemistry from Yale and is currently a professor in the biomedical engineering department and medical physics department at the University of Wisconsin-Madison. He is a member of both OSA and SPIE and is an editor for Journal of Biomedical Optics.

\section{Competing interests}

The authors declare that they have no competing interests.

\section{Ethics approval and consent to participate}

The University of Wisconsin Madison IRB reviewed and approved the use of consenting patients (written) under studies 2011-0255 and 2014-1223.

\section{Author details}

'Laboratory for Optical and Computational Instrumentation, Department of Biomedical Engineering, University of Wisconsin - Madison, 1550 Engineering Drive, Madison, WI 53706, USA. ${ }^{2}$ Medical Physics Department, University of Wisconsin - Madison, 1111 Highland Avenue, Madison, WI 53706, USA. ${ }^{3}$ Morgridge Institute for Research, 330 N. Orchard Street, Madison, WI 53715, USA. ${ }^{4}$ Department of Obstetrics and Gynecology, University of Wisconsin - Madison, 600 Highland Avenue, Madison, WI 53706, USA.

Received: 25 May 2016 Accepted: 26 January 2017

Published online: 06 February 2017

\section{References}

1. Iyer VR, Lee SI. MRI, CT, and PET/CT for ovarian cancer detection and adnexal lesion characterization. AJR Am J Roentgenol. 2010;194(2):311-21.

2. Fishman DA, Cohen L, Blank SV, Shulman L, Singh D, Bozorgi K, Tamura R, Timor-Tritsch I, Schwartz PE. The role of ultrasound evaluation in the detection of early-stage epithelial ovarian cancer. Am J Obstet Gynecol. 2005;192(4):1214-21. discussion 1221-1212.

3. van Nagell Jr JR, DePriest PD, Reedy MB, Gallion HH, Ueland FR, Pavlik EJ, Kryscio RJ. The efficacy of transvaginal sonographic screening in asymptomatic women at risk for ovarian cancer. Gynecol Oncol. 2000;77(3):350-6.

4. Bowtell DD. The genesis and evolution of high-grade serous ovarian cancer. Nat Rev Cancer. 2010;10(11):803-8.

5. Prat J. New insights into ovarian cancer pathology. Ann Oncol. 2012;23 Suppl 10:x111-117.

6. Kurman RJ, Visvanathan K, Roden R, Wu TC, Shih le M. Early detection and treatment of ovarian cancer: shifting from early stage to minimal volume of disease based on a new model of carcinogenesis. Am J Obstet Gynecol. 2008;198(4):351-6.

7. Przybycin CG, Kurman RJ, Ronnett BM, Shih le M, Vang R. Are all pelvic (nonuterine) serous carcinomas of tubal origin? Am J Surg Pathol. 2010; 34(10):1407-16.

8. Ricciardelli C, Rodgers RJ. Extracellular matrix of ovarian tumors. Semin Reprod Med. 2006;24(4):270-82.

9. Kauppila S, Bode MK, Stenback F, Risteli L, Risteli J. Cross-linked telopeptides of type I and III collagens in malignant ovarian tumours in vivo. Br J Cancer. 1999;81(4):654-61.

10. Chen X, Nadiarynkh O, Plotnikov S, Campagnola PJ. Second harmonic generation microscopy for quantitative analysis of collagen fibrillar structure. Nat Protoc. 2012;7(4):654-69.

11. Campagnola PJ, Millard AC, Terasaki M, Hoppe PE, Malone CJ, Mohler WA. 3-Dimesional High-Resolution Second Harmonic Generation Imaging of Endogenous Structural Proteins in Biological Tissues. Biophys J. 2002;82:493-508.

12. Watson JM, Rice PF, Marion SL, Brewer MA, Davis JR, Rodriguez JJ, Utzinger U, Hoyer PB, Barton JK. Analysis of second-harmonic-generation microscopy in a mouse model of ovarian carcinoma. J Biomed Opt. 2012;17(7):076002.

13. Adur J, Pelegati VB, de Thomaz AA, Baratti MO, Andrade LA, Carvalho HF, Bottcher-Luiz F, Cesar CL. Second harmonic generation microscopy as a 
powerful diagnostic imaging modality for human ovarian cancer. J Biophotonics. 2014;7(1-2):37-48.

14. Wen BL, Brewer MA, Nadiarnykh O, Hocker J, Singh V, Mackie TR, Campagnola PJ. Texture analysis applied to second harmonic generation image data for ovarian cancer classification. J Biomed Opt. 2014;19(9): 096007.

15. Williams RM, Flesken-Nikitin A, Ellenson LH, Connolly DC, Hamilton TC, Nikitin AY, Zipfel WR. Strategies for high-resolution imaging of epithelial ovarian cancer by laparoscopic nonlinear microscopy. Transl Oncol. 2010;3(3):181-94.

16. Nadiarnykh O, LaComb RB, Brewer MA, Campagnola PJ. Alterations of the extracellular matrix in ovarian cancer studied by Second Harmonic Generation imaging microscopy. BMC Cancer. 2010;10:94.

17. Lacomb R, Nadiarnykh O, Townsend SS, Campagnola PJ. Phase Matching considerations in Second Harmonic Generation from tissues: Effects on emission directionality, conversion efficiency and observed morphology. Optics Comm. 2008;281:1823-32.

18. Liu Y, Brand RE, Turzhitsky V, Kim YL, Roy HK, Hasabou N, Sturgis C, Shah D, Hall C, Backman V. Optical markers in duodenal mucosa predict the presence of pancreatic cancer. Clin Cancer Res. 2007;13(15 Pt 1):4392-9.

19. Roy HK, Liu Y, Wali RK, Kim YL, Kromine AK, Goldberg MJ, Backman V. Fourdimensional elastic light-scattering fingerprints as preneoplastic markers in the rat model of colon carcinogenesis. Gastroenterology. 2004;126(4):107181; discussion 948.

20. Schindelin J, Arganda-Carreras I, Frise E, Kaynig V, Longair M, Pietzsch T, Preibisch S, Rueden C, Saalfeld S, Schmid B, et al. Fiji: an open-source platform for biological-image analysis. Nat Methods. 2012;9(7):676-82.

21. Wang L, Jacques SL, Zheng L. MCML-Monte Carlo modeling of light transport in multi-layered tissues. Comput Methods Programs Biomed. 1995:47(2):131-46.

22. Lacomb R, Nadiarnykh O, Campagnola PJ. Quantitative SHG imaging of the diseased state Osteogenesis Imperfecta: Experiment and Simulation. Biophys J. 2008:94:4504-14.

23. Hall G, Eliceiri KW, Campagnola PJ. Simultaneous determination of the second-harmonic generation emission directionality and reduced scattering coefficient from three-dimensional imaging of thick tissues. J Biomed Opt. 2013;18(11):116008.

24. Li H, Xie S. Measurement method of the refractive index of biotissue by total internal reflection. Appl Opt. 1996;35:1793-5.

25. Hall G, Jacques SL, Eliceiri KW, Campagnola PJ. Goniometric measurements of thick tissue using Monte Carlo simulations to obtain the single scattering anisotropy coefficient. Biomedical optics express. 2012;3(11):2707-19.

26. Rogers JD, Capoglu IR, Backman V. Nonscalar elastic light scattering from continuous random media in the Born approximation. Opt Lett. 2009;34(12):1891-3.

27. Turzhitsky V, Radosevich A, Rogers JD, Taflove A, Backman V. A predictive model of backscattering at subdiffusion length scales. Biomed Opt Express. 2010;1(3):1034-46

28. Mertz J, Moreaux L. Second-harmonic generation by focused excitation of inhomogeneously distributed scatterers. Opt Commun. 2001;196(1-6):325-30.

29. Rieber A, Nussle K, Stohr I, Grab D, Fenchel S, Kreienberg R, Reske SN, Brambs HJ. Preoperative diagnosis of ovarian tumors with MR imaging: comparison with transvaginal sonography, positron emission tomography, and histologic findings. AJR Am J Roentgenol. 2001;177(1):123-9.

30. Hall G, Tilbury KB, Campbell KR, Eliceiri KW, Campagnola PJ. Experimental and simulation study of the wavelength dependent second harmonic generation of collagen in scattering tissues. Opt Lett. 2014;39(7):1897-900.

31. Nadiarnykh O, Plotnikov S, Mohler WA, Kalajzic I, Redford-Badwal D, Campagnola PJ. Second Harmonic Generation imaging microscopy studies of Osteogenesis Imperfecta. JBiomed Opt. 2007;12:051805.

\section{Submit your next manuscript to BioMed Central and we will help you at every step:}

- We accept pre-submission inquiries

- Our selector tool helps you to find the most relevant journal

- We provide round the clock customer support

- Convenient online submission

- Thorough peer review

- Inclusion in PubMed and all major indexing services

- Maximum visibility for your research

Submit your manuscript at www.biomedcentral.com/submit

\section{O) Biomed Central}

